\title{
Cerebrotendinous xanthomatosis with progressive cerebellar vacuolation
}

\author{
Six-year MRI follow-up
}

\author{
Andrea Mignarri • Maria Teresa Dotti • \\ Marina Del Puppo • Gian Nicola Gallus • \\ Antonio Giorgio • Alfonso Cerase • Lucia Monti
}

Received: 27 February 2012 / Accepted: 5 March 2012 / Published online: 15 March 2012

(C) Springer-Verlag 2012

\section{Dear Sir,}

Cerebrotendinous xanthomatosis (CTX) is a rare autosomal recessive lipid storage disease with deposition of cholestanol in many tissues causing both systemic and neurological involvement. CTX is due to mutations in the CYP27A1 gene leading to defective activity of sterol 27-hydroxylase. Therapy with chenodeoxycholic acid (CDCA) normalizes bile acids synthesis and prevents clinical deterioration. Brain magnetic resonance imaging (MRI) typically includes dentate nuclei hyperintensities on T2-weighted and FLAIR sequences, and has been reported to be substantially stable after treatment with CDCA [1,2].

We report on a 33-year-old woman with gait unsteadiness since the age of 30 years. Her history revealed mental retardation, diarrhea and cataracts. Clinical examination disclosed dysarthria and ataxia. Tendon xanthomas were absent. Biochemical analysis revealed elevated plasma levels of cholestanol and $7 \alpha$-hydroxy-4-cholesten-3-one, and decreased 27-hydroxycholesterol (27OHC) concentration. CYP27A1 gene analysis uncovered the $752 \mathrm{C}>\mathrm{A}$ homozygous mutation. Therapy with CDCA was started and the

A. Mignarri $\cdot$ M. T. Dotti $(\triangle) \cdot$ G. N. Gallus $\cdot$ A. Giorgio

Department of Neurological and Behavioural Sciences,

University of Siena,

Viale Bracci 2,

53100 Siena, Italy

e-mail: dotti@unisi.it

M. Del Puppo

Department of Experimental Medicine,

University of Milano-Bicocca,

Milano, Italy

A. Cerase $\cdot$ L. Monti

Unit of Diagnostic and Therapeutic Neuroradiology, AOUS,

Siena, Italy patient periodically re-evaluated. Tendon xanthomas never emerged, and diarrhea disappeared. By contrast, ataxia worsened over time (Fig. 1a). A sharp reduction of cholestanol and $7 \alpha$-hydroxy-4-cholesten-3-one serum levels was found (Fig. 1b, c), while 27OHC still showed very low concentrations without substantial changes from baseline.

Brain MRI at $1.5 \mathrm{~T}$ was performed every 2 years until the age of 39 years together with clinical and biochemical follow-up. At baseline, T2-weighted and FLAIR hyperintensities in the dentate nuclei and surrounding white matter (WM) were evident. Areas of hypointensity on both T1-weighted and FLAIR images were found within cerebellar lesions. Computed tomography, and susceptibility weighted and T2*-weighted MRI ruled out the presence of calcifications. Six-year MRI follow-up showed on FLAIR images increase of the hypointense areas, which replaced hyperintensities suggesting vacuolation (Fig. 1d). MR spectroscopy (MRS) on the cerebellar hemispheres (Fig. 2) revealed the following: i) diffuse decrease of $\mathrm{N}$-acetylaspartate (NAA)/creatine $(\mathrm{Cr})=1.58$ (n.v. $2.27 \pm 0.29$ ) and choline $(\mathrm{Cho}) / \mathrm{Cr}=0.53$ (n.v. $0.78 \pm 0.04)$, and increased myoinositol $(\mathrm{mI}) / \mathrm{Cr}$ ratio, $\mathrm{mI} / \mathrm{Cr}=0.43$ (n.v. $0.27 \pm 0.03$ ) in normal appearing areas; ii) lactate (Lac) and lipids (Lip) peaks in the voxels of the FLAIRhyperintense areas surrounding the FLAIR-hypointense lesions; and iii) very high Lac and Lip peaks and marked NAA/Cr decrease in the FLAIR-hypointense lesions.

The serial evaluation of clinical, biochemical and MRI findings in this patient revealed that CDCA therapy was effective in decreasing serum levels of bile acids intermediates and stabilizing systemic manifestations, but failed to prevent worsening of neurological disturbances and brain lesions. The lack of correspondence between neurological and biochemical data is a crucial point, which remains open to a wide range of interpretations. 


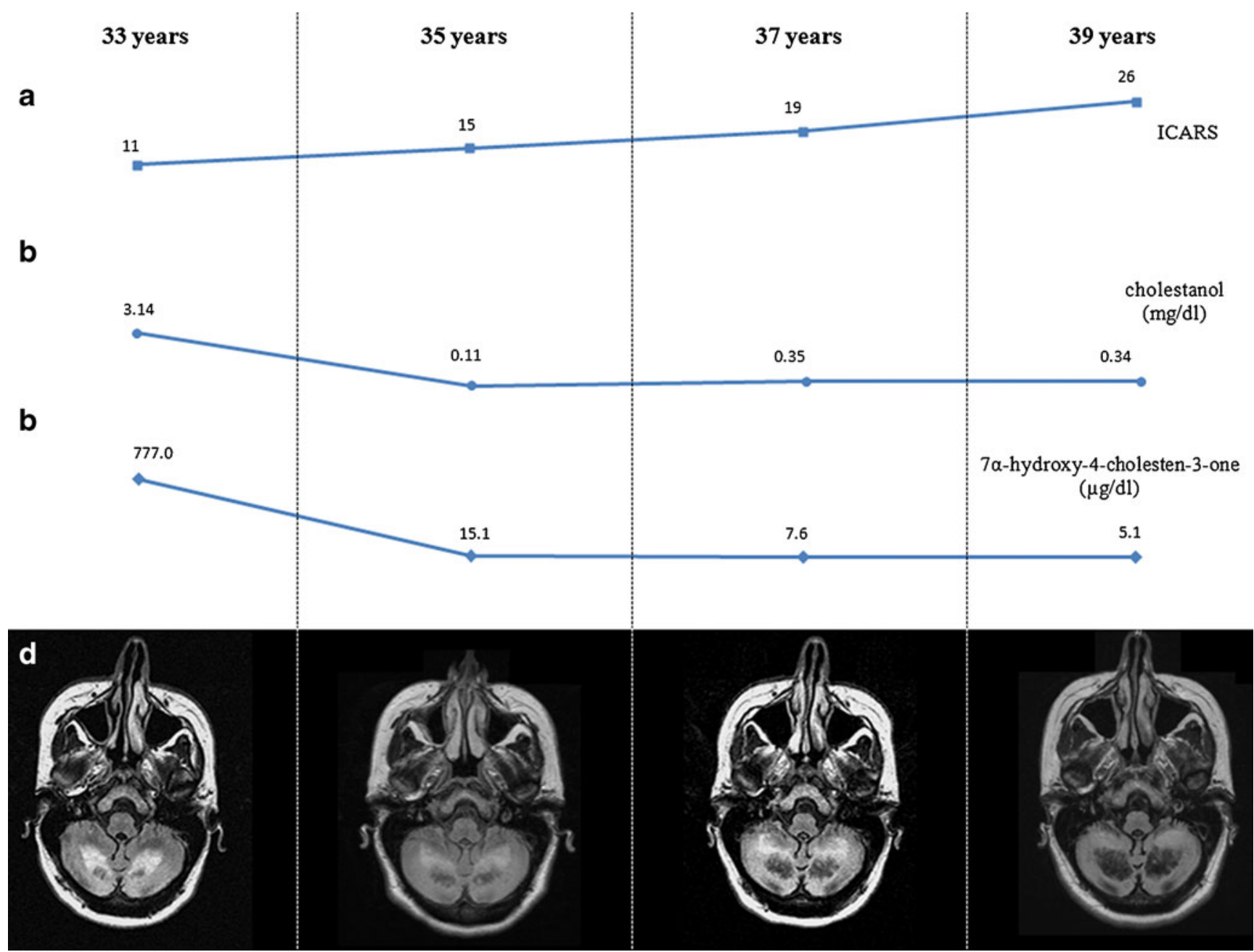

Fig. 1 Six-year clinical, biochemical and brain MRI follow-up. a International co-operative ataxia rating scale (ICARS) score increases over time. b Serum cholestanol levels (n.v. $0.34 \pm 0.16 \mathrm{mg} / \mathrm{dl}$ ). c Serum

Mechanisms of neurotoxicity in CTX are still unclear and seem to go beyond the concentration-dependent accumulation of cholestanol. Brain cholestanol may originate from $7 \alpha$-hydroxylated precursors such as $7 \alpha$-hydroxy-4-cholesten-3-one, which crosses the blood-brain barrier more efficiently and can be converted to cholestanol in neuronal and glial cells [3]. In our patient, CDCA administration led to normalization of cholestanol and marked decrease of $7 \alpha$ hydroxy-4-cholesten-3-one, whose levels remained slightly elevated. The role of prolonged exposure to mildly increased serum levels of $7 \alpha$-hydroxy-4-cholesten-3-one in neurotoxicity needs further investigation.

The lack of $27 \mathrm{OHC}$ could also play a pathogenetic role. Cerebral $27 \mathrm{OHC}$ mostly originates from the circulation and represents an important regulator of cholesterol metabolism in the brain [4]. In our case, CDCA was ineffective in restoring plasma $27 \mathrm{OHC}$. Further studies are warranted to understand if $27 \mathrm{OHC}$ deficiency causes an alteration of brain cholesterol homeostasis.
$7 \alpha$-hydroxy-4-cholesten-3-one levels (n.v. $1.5 \pm 0.4 \mu \mathrm{g} / \mathrm{dl}$ ). d FLAIR axial MRI shows increased extension of WM hyperintensities and hypointense areas, which replace hyperintensities over time

If so, disregulation of the oxysterols may be considered as a target for new therapies.

The presence of cerebellar hypointensities with "cystic" appearance on T1-weighted and FLAIR sequences has been reported in another CTX subject and considered as an unusual finding [5]. The cerebellar lesions on FLAIR images followed over time in our study might represent "two sides of the same coin": while hyperintensities are probably the initial result of abnormal lipid storage, hypointense areas may be due to cerebellar degeneration caused by cholestanolinduced apoptosis [6] or by other yet undefined mechanisms leading to neuroaxonal loss.

MRS findings add relevant metabolic information: the elevated $\mathrm{mI} / \mathrm{Cr}$ ratio could be correlated to increased glial cell population, and both Lac and Lip peaks on the border of the vacuolating lesions may be the expression of a critical metabolic condition preceding apoptosis. Their possible utilization as a spectroscopic marker of disease evolution deserves further studies. 


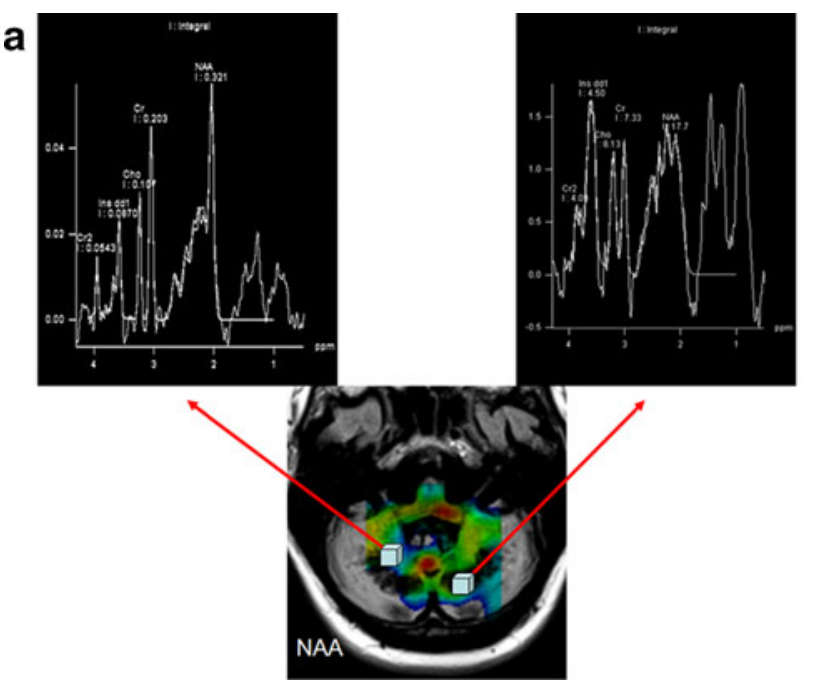

Fig. 2 Cerebellar MRS imaging performed at age 39 years. a Right hemisphere: Lip and Lac peaks in the parenchyma surrounding the FLAIR-hypointense lesion. Left hemisphere: increased Lip and Lac and decreased NAA peaks in the FLAIR-hypointense lesion. NAA

In conclusion, our serial evaluation in a CTX patient with progressive ataxia revealed a peculiar MRI pattern, consistent with cerebellar vacuolation, which could be a marker of poor response to CDCA treatment.

Conflict of interest We declare that we have no conflict of interest.

\section{References}

1. Barkhof F, Verrips A, Wesseling P, van Der Knaap MS, van Engelen BG, Gabreëls FJ, Keyser A, Wevers RA, Valk J (2000) Cerebrotendinous xanthomatosis: the spectrum of imaging findings and the correlation with neuropathologic findings. Radiology 217:869-876 b

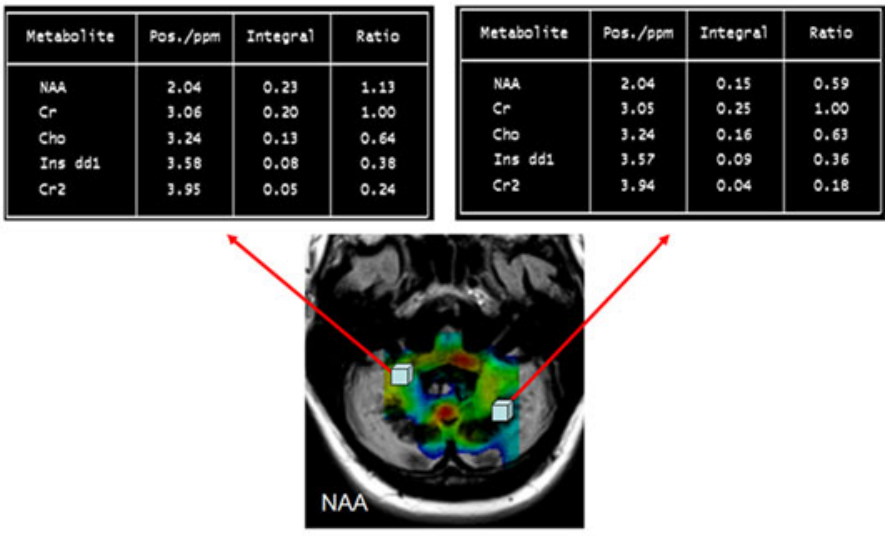

parametric map: lower (green and blue) and higher (yellow and red) concentrations. b Right hemisphere: low NAA/Cr in normal appearing WM; left hemisphere: marked NAA/Cr decrease on FLAIRhypointense lesion

2. De Stefano N, Dotti MT, Mortilla M, Federico A (2001) Magnetic resonance imaging and spectroscopic changes in brains of patients with cerebrotendinous xanthomatosis. Brain 124:121-131

3. Panzenboeck U, Andersson U, Hansson M, Sattler W, Meaney S, Björkhem I (2007) On the mechanism of cerebral accumulation of cholestanol in patients with cerebrotendinous xanthomatosis. J Lipid Res 48:1167-1174

4. Björkhem I, Cedazo-Minguez A, Leoni V, Meaney S (2009) Oxysterols and neurodegenerative diseases. Mol Aspects Med 30:171179

5. Androdias G, Vukusic S, Gignoux L, Boespflug-Tanguy O, Acquaviva C, Zabot MT, Couvert P, Carrie A, Confavreux C, Labauge P (2012) Leukodystrophy with a cerebellar cystic aspect and intracranial atherosclerosis: an atypical presentation of cerebrotendinous xanthomatosis. J Neurol 259:364-366

6. Inoue K, Kubota S, Seyama Y (1999) Cholestanol induces apoptosis of cerebellar neuronal cells. Biochem Biophys Res Commun 256:198-203 